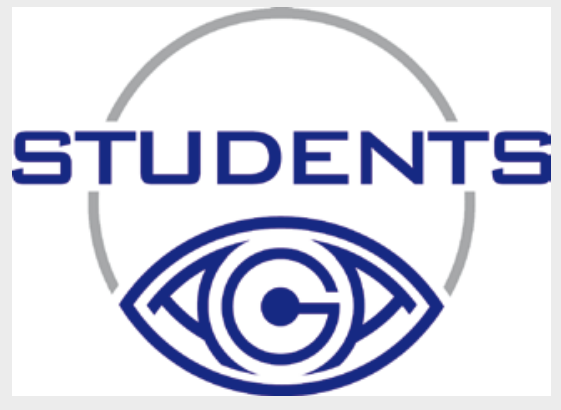

\section{Impressum}

Andreas Voss

Abteilung und Poliklinik für

Sportorthopädie

Klinikum rechts der Isar

Technische Universität München

Ismaninger Str. 69

D-81675 München

a.voss@tum.de

Arthroskopie 2015 - 28:157-159

DOI 10.1007/s00142-015-0002-9

(C) Springer-Verlag Berlin Heidelberg 2015

\section{Redaktion}

Prof. Dr. Andreas Imhoff

Andreas Voss

\title{
Bericht zur 2. AGA Studenten Advanced School vom 23. bis 24. 1.2015 in München
}

Am 23. und 24.1.2015 fand die 2. AGA Studenten Advanced School unter der wissenschaftlichen Leitung von Prof. Dr. Andreas B. Imhoff in der anatomischen Anstalt der Ludwig-Maximillian-Universität München statt. Nach dem Erfolg des letzten Jahres lag auch dieses Jahr der Schwerpunkt auf der operativen Anatomie und den offenen Zugangswegen von Schulter- und Kniegelenk.

Zwölf AGA-Studenten der letzten Summer-School in Basel wurden auf Basis des neuen Curriculums ausgewählt, diesen Kurs zu absolvieren. Den Teilnehmern bot sich in einem zwei- tägigen Intensiv-Workshop die Möglichkeit, die operativen $\mathrm{Zu}$ gangswege an humanen Schulter- und Kniegelenkspräparaten $\mathrm{zu}$ erforschen und die Anatomie aus der Perspektive der Chirurgie zu verstehen.

Der erste Tag stand ganz im Zeichen des Schultergelenks. Am Vormittag wurden Vorträge zur operativen Anatomie mit dem Fokus auf die angrenzenden Nerven und Gefäße angeboten. Des Weiteren erläuterten und diskutierten die Referenten drei verschiedene operative $\mathrm{Zu}$ gänge (deltoideopectoral, lateral, posterior) und deren Indikationen. Am Nachmittag konnten die AGA-Studenten unter Anleitung der erfahrenen Instruktoren eigene Praxiserfahrungen an Kadaverschultern sammeln ( Abb. 1). Durch die ausgezeichnete Betreuung durch die Spezialisten blieb keine Frage unbeantwortet und die großzügig bemessene Zeit konnte effektiv genutzt werden. Der Tag klang in einem vietnamesischen Restaurant in der Münchner Innenstadt aus.

Der zweite Tag widmete sich dem Kniegelenk. Wie am Vortag wurden zunächst die theoretischen Grundlagen zur operativen Anatomie und den Zugangswegen am Kniegelenk vermittelt. Die Referenten erläuterten die genauen operativen Abläufe im Detail und erörterten potenzielle intraoperative Fehlerquellen und Komplikationen. Nach den lehrreichen Vorträgen wurden die Kniegelenke präpariert. Die Zugänge (anteromedial, postero- medial, lateral und posterior) konnten unter Anleitung erlernt und die Strukturen rund um das Kniegelenk dargestellt werden.

Dank der Firma MEDI hatten die Studenten ebenfalls die Möglichkeit, sich über die neuesten Schulter- und Knieorthesen zu informieren, diese selbst anzulegen und zu probieren.

Zusammenfassend war die zweite AGA Advanced-School wieder ein voller Erfolg (- Abb. 2). Dies zeigte vor allem die exzellente Bewertung der Teilnehmer im abschließenden Evaluationsbogen mit einer Gesamtnote von 1,1. Die Studenten lernten die Gelenksanatomie und -Chirurgie in kleinen Gruppen unter der Anleitung von renommierten Experten kennen. Die selbstständige Präparation gewährte neue Einblicke in die angrenzenden und zu schonenden Strukturen der Operationsgebiete, welche im konventionellen

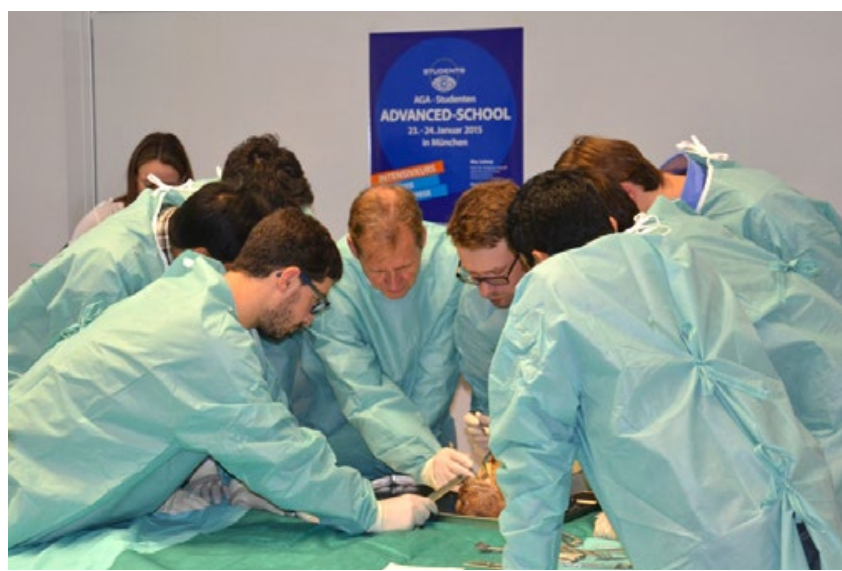

Abb. $1 \triangle$ Prof. Dr. Imhoff erklärt die Zugangswege der Schulter am humanen Präparat

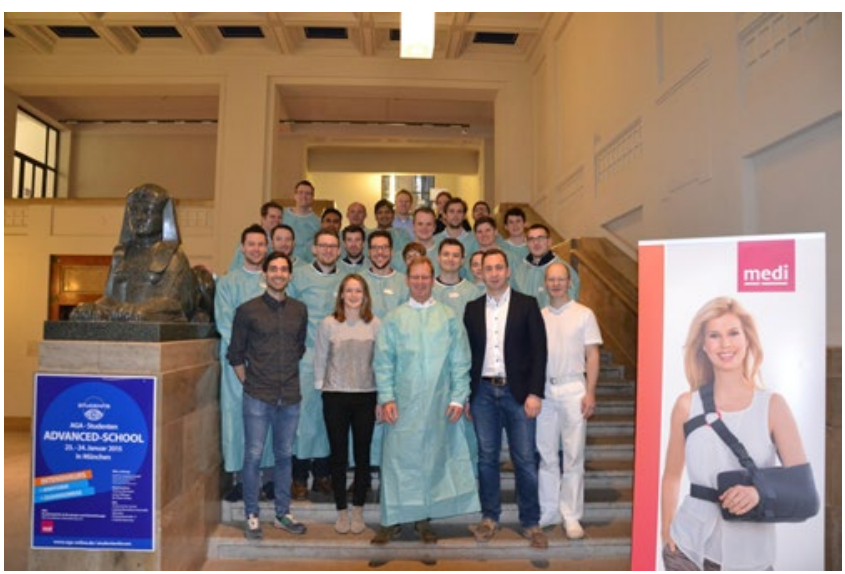

Abb. 2 Das Gruppenfoto der 2. AGA Studenten Advanced School in der anatomischen Anstalt der LMU München 
Anatomiekurs nicht vermittelt werden. In einer kollegialen Atmosphäre konnte ein reger Austausch zwischen Teilnehmern und Instruktoren stattfinden.

Ein besonderer Dank gilt Prof. Dr. Imhoff, allen Referenten und der Firma MEDI, durch die die zweite Advanced-School zu einem beispielhaften Projekt der AGA-Studenten werden konnte.

Eure Organisatoren

Lukas, Theresa und Elmar

\section{Arthroskopiekurs in Arosa vom 18. bis 23.1.2015}

Auf Einladung von Prof. Dr. Strobel und Dr. Birkner durfte ich vom 18. Bis 23.1.2015 am Arthroskopiekurs in Arosa kostenfrei teilnehmen. Vormittags

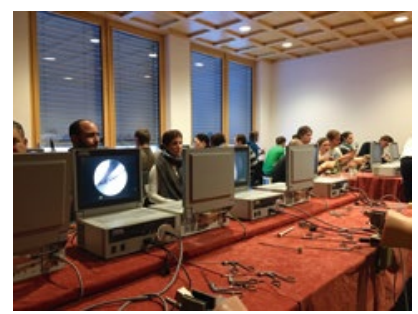

Abb. $1 \triangle$ Umsetzung der Meniskuschirurgie am Model

wurden klinisch relevante Themen der Arthroskopie besprochen, nachmittags wurde mit Bereitstellung der nötigen Modelle u.a. Techniken der Meniskusrefixationen und -resektionen erlernt ( Abb. 1). Der

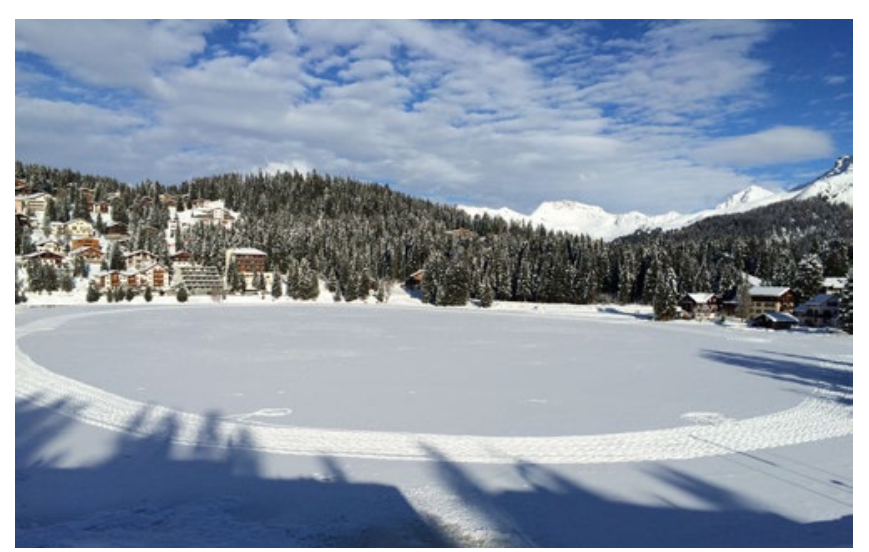

Abb. $2 \triangle$ Blick auf Arosa
Hüttenabend mit einer außergewöhnlichen Schlittenfahrt war ein Höhepunkt der Woche. Mit einer gemeinsamen Abschlussveranstaltung endete schließlich diese unvergessliche Woche (Abb. 2). Ich habe von dieser Lehrveranstaltung sehr profitiert und möchte mich nochmals bei Prof. Dr. Strobel und Dr. Birkner ganz herzlich bedanken, mir dies ermöglicht zu haben.

(Einen ausführlicheren Bericht von der Veranstaltung können Sie auf der AGA-Web-Seite nachlesen: www.aga-online.de/ studentenforum)

\section{Christina Kahlert}

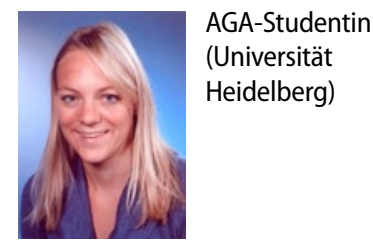




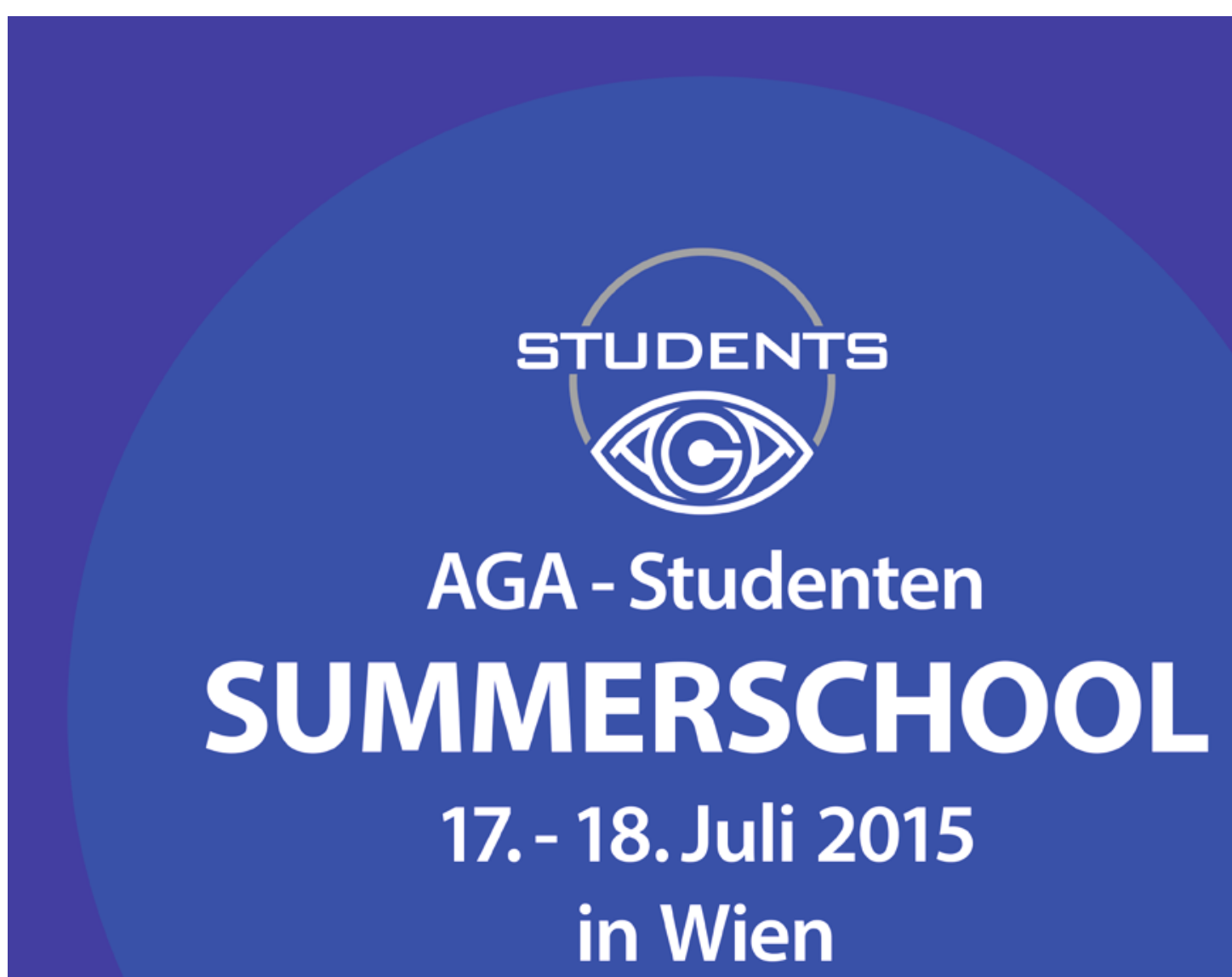

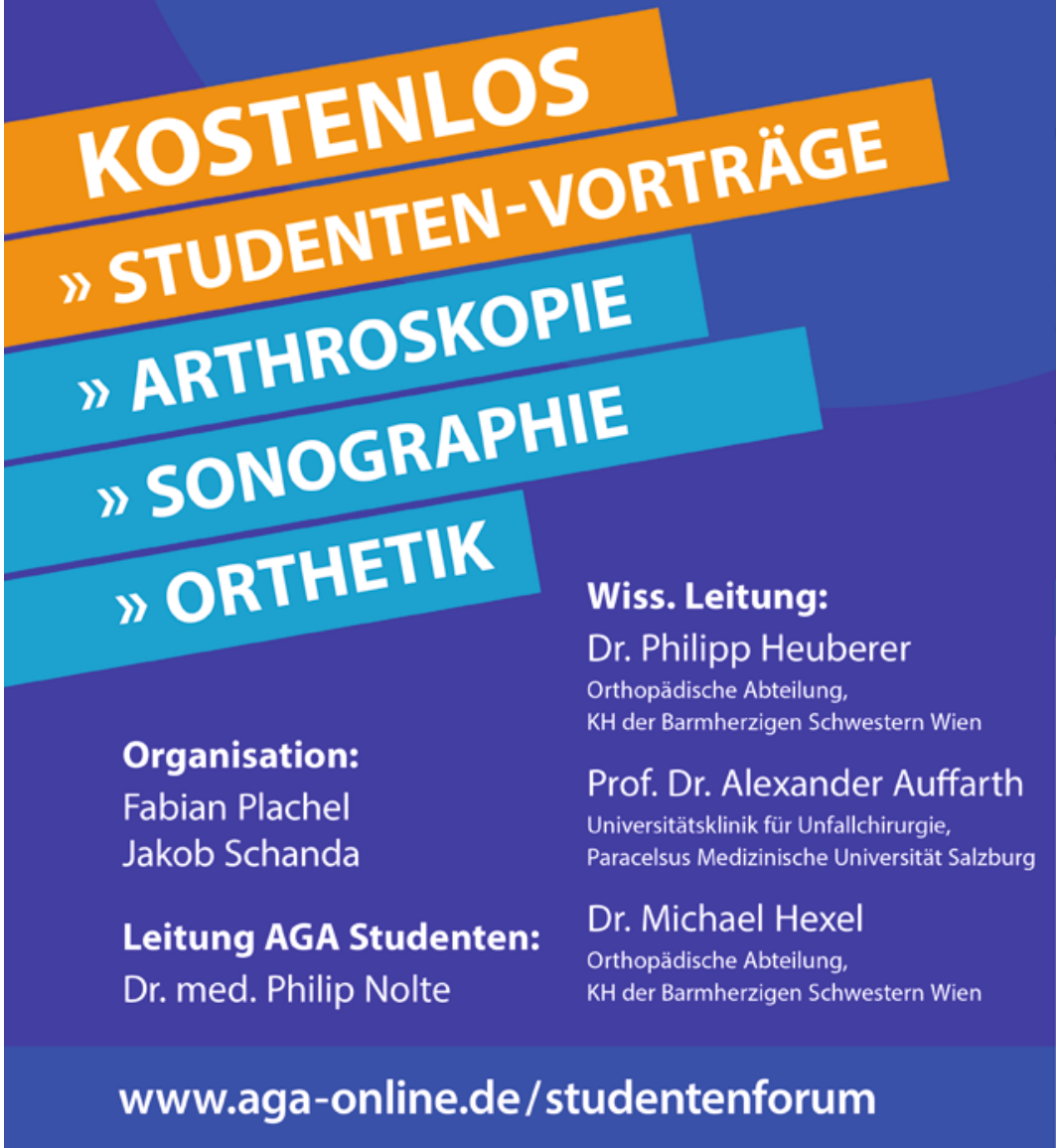

Ort:

Krankenhaus der

Barmherzigen Schwestern

Stumpergasse 13

A-1060 Wien

\section{AGA}

Gesellschaft für Arthroskopie und Gelenkchirurgie

Mit freundlicher Unterstützung von:

ottobock.

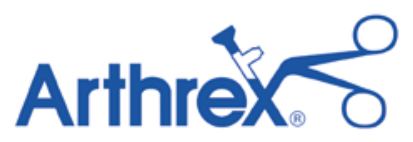

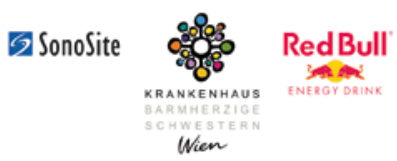

Vorläufiger Stand 09.03.2015 
Hier steht eine Anzeige.

算 Springer 